\title{
Imaging clathrin mediated endocytosis in action.
}

\author{
Christien J. Merrifield*, Morris Feldman**, Wolfhard Almers***. \\ * Post Doctoral Fellow, Department of Cellular and Molecular Physiology, Yale \\ University School of Medicine, 333 Cedar Street, SHM-B103, Newhaven, CT, 06520 \\ ** Research Assistant, Department of Biomedical Sciences, Cornell University, Ithaca, \\ New York, 14853 \\ *** Senior Scientist, Vollum Institute, Oregon Health and Sciences University, Portland, \\ OR, 97201
}

Clathrin mediated endocytosis is one of the principle means of receptor internalization in eukaryotic cells. In this mechanism receptors concentrate in specialized protein lattices at the plasma membrane known as clathrin coated pits. Invagination and budding of receptor laden coated pits leads to the formation of clathrin coated vesicles. These vesicles subsequently uncoat, and carry their receptor cargo further into the cell for processing (reviewed in $[1,2]$ ).

While a dozen or more proteins have been implicated in clathrin mediated endocytosis, it is unclear how these components are temporally and spatially orchestrated in living cells to achieve vesicle fission. To address this problem we have used two-color evanescent field microscopy to simultaneously image clathrin coated pits (labeled red) and a second protein of interest (labeled green) in living 3T3 fibroblasts.

Evanescent field, or total internal reflection (TIRF), microscopy is a specialized form of fluorescence microscopy that relies on the total internal reflection of excitation light at a glass/water interface to generate a sub-wavelength sized evanescent field. This evanescent field is used to selectively excite fluorophores within $\sim 100 \mathrm{~nm}$ of the surface of the glass. For a cell biologist this provides a convenient means of imaging fluorophores at the lower surface of cells adhering to a high refractive index glass coverslip [3].

We used a custom built two-color evanescent field microscopy to investigate the recruitment and dismissal of GFP-labeled proteins at clathrin coated pits in live 3T3 fibroblasts. Cells expressing clathrin light chain a-DsRed (clathrin-DsRed) were transiently transfected with one of a variety of GFP-conjugated proteins. Doubly transfected cells were plated onto high refractive index glass coverslips and images acquired at $0.5 \mathrm{~Hz}$ at $28 \mathrm{C}$. Images were either acquired using either EF illumination alone or alternating evanescent field and epifluorescence (Epi) illumination [4].

The lower surface 3T3 fibroblasts expressing clathrin-DsRed were covered in numerous small fluorescent dots representing clathrin-coated structures (CCSs) that dimmed under EF illumination after 1-2 minutes of residence near the plasma membrane. When EF and Epi illumination alternated, dimming occurred under EF but not Epi. Epi illumination 
floods the entire cell with excitation light while EF illumination only excites fluorophores within the first 100nm of the cell. Therefore if a CCS disappears under EF, but not Epi, illumination this must represent genuine CCS internalization. The majority $(>80 \%)$ of CCSs which disappear under EF illumination genuinely internalize.

Using disappearance of CCSs under EF illumination as a signature for internalization we explored the recruitment of a variety of GFP-labeled proteins to CCSs. Transferrin receptor has often been used as a model receptor cargo for clathrin mediated endocytosis. Transferrin receptor-GFP was found to be present in CCSs at all times and internalized simultaneously with clathrin. In contrast, the GTPase dynamin I was recruited to relatively immobile CCSs at low levels, but its concentration raised 3 fold just prior to CCS internalization. Internalization of CCSs coincided with a concomitant drop in dynamin I concentration. During internalization a sub-micron plume of $\beta$-actin formed, possibly helping to drive the inward movement of the CCSs. The accumulation of $\beta$-actin probably represents polymerization into filamentous actin since several proteins involved in actin polymerization were recruited at or near to CCSs as they internalized. These included neural Wiskott-Aldrich syndrome protein (N-WASP), mouse actin binding protein 1 (mAbp1), syndapin 1 and actin related protein 3 (Arp3). Our findings are consistent with the predicted function(s) in clathrin mediated endocytosis of several of the proteins which we have seen recruited to internalizing CCSs.

We suggest that internalization of CCSs is triggered by a critical event in endocytosis (such as the accumulation of dynamin) that initiates a burst of actin polymerization through a pathway that includes N-WASP, syndapin I, mAbp1 and Arp3. We conclude that dynamin accumulation may provide the trigger, and actin polymerization may provide the force for CCS movement away from the cell surface.

[1] Mukherjee S, Gosh RN, Maxfield FR, Physiol Rev. (1997) Jul;77(3):759-803

[2] Higgins MK, McMahon HT, Trends Biochem Sci. (2002) May; 27(5):257-63

[3] Steyer JA, Almers W, Nat Rev Mol Cell Biol. (2001) Apr;2(4):268-75

[4] Merrifield CJ, Feldman ME, Wan L, Nat Cell Biol. (2002) Sep;4(9):691-8 